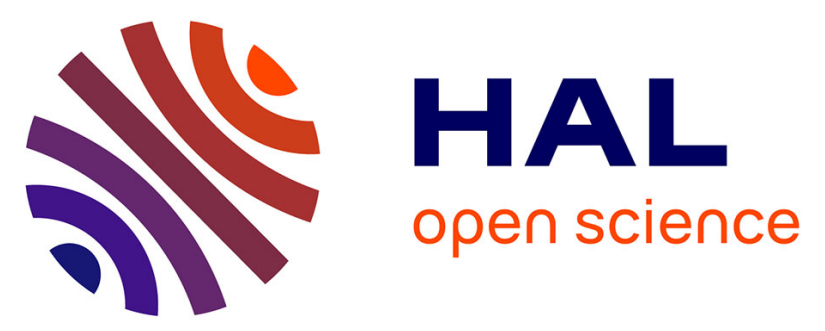

\title{
Analysis of the trade-off between resolution and bandwidth for a nanoforce sensor based on diamagnetic levitation.
}

\author{
Emmanuel Piat, Joël Abadie, Stéphane Oster
}

\section{- To cite this version:}

Emmanuel Piat, Joël Abadie, Stéphane Oster. Analysis of the trade-off between resolution and bandwidth for a nanoforce sensor based on diamagnetic levitation.. 2012 IEEE International Conference on Robotics and Automation (ICRA 2012), May 2012, Saint Paul, MN United States. pp.1416-1421, 10.1109/ICRA.2012.6224845 . hal-01313515

\section{HAL Id: hal-01313515 \\ https://hal.science/hal-01313515}

Submitted on 10 May 2016

HAL is a multi-disciplinary open access archive for the deposit and dissemination of scientific research documents, whether they are published or not. The documents may come from teaching and research institutions in France or abroad, or from public or private research centers.
L'archive ouverte pluridisciplinaire HAL, est destinée au dépôt et à la diffusion de documents scientifiques de niveau recherche, publiés ou non, émanant des établissements d'enseignement et de recherche français ou étrangers, des laboratoires publics ou privés. 


\title{
Analysis of the trade-off between resolution and bandwidth for a nanoforce sensor based on diamagnetic levitation
}

\author{
Emmanuel Piat and Joël Abadie and Stéphane Oster
}

\begin{abstract}
Nanoforce sensors based on passive diamagnetic levitation with a macroscopic seismic mass are a possible alternative to classical Atomic Force Microscopes when the force bandwidth to be measured is limited to a few Hertz. When an external unknown force is applied to the levitating seismic mass, this one acts as a transducer that converts this unknown input into a displacement that is the measured output signal. Because the inertia effect due to the mass of such macroscopic transducers can not be neglected for timevarying force measurement, it is necessary to deconvolve the displacement to correctly estimate the unknown input force. A deconvolution approach based on a Kalman filter and controlled by a scalar parameter has been recently proposed. The adjustement of this parameter leads to a trade-off that is analysed in this paper in term of resolution and bandwidth of the estimated force. Associated tools to help the end-user to set this parameter are also described.
\end{abstract}

\section{INTRODUCTION}

All micro or nanoforce sensors use a transducer to convert the force into a measurable effect that is most of the time related to a displacement $x$. For linear sensors, the usual scalar expression used to calculate the component $F$ of the applied force $\vec{F}$ in one-direction $\vec{x}$ of space simply consists in:

$$
F=K x \quad K>0
$$

$K$ is the mechanical stiffness of the transducer along $\vec{x}$ (by convention $x$ is set to zero when there is no displacement). This steady-state equation supposes that the dynamic response of the transducer can be neglected. This is usually considered to be the case for classical designs using monolithic elastic microstructures such as microcantilevers which have a high resonant frequency: AFM based microforce sensors [1] [2], piezoresistive microforce sensors [3], capacitive microforce sensors [4], piezoelectric microforce sensors [5], etc. When the transient dynamic of the transducer due to the evolution of the successive derivatives of $x$ is not negligible, (1) can not be used and the force estimation becomes an open-loop deconvolution problematic of a noisy output signal that is shown in the Figure 1. This problematic also exists in closed-loop force sensor based on seismic mass [6]. In this case, the inputs of the transducer are the unknown force like previously and the controller ouput. As the latter is known, this does not change the problematic addressed here that is also related to unknown input observer (UIO) and is common in control engineering or robotics every time a

FEMTO-ST Institute

CNRS - UFC - ENSMM - UTBM

Besançon - France

epiat@ens 2 m.fr

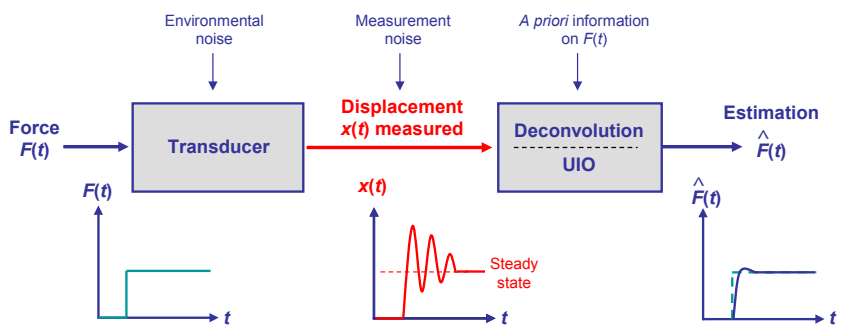

Fig. 1. Force estimation using a deconvolution approach or an unknown input observer (UIO).

unknown input signal must be determined in open or closedloop systems with noisy ouputs. Nevertheless, it has been little addressed in the specific context of micro or nanoforce measurement in microrobotics. The UIO design proposed in [7] requires to set several parameters and the level of noise in the force estimation can't be directly adjusted. Another approach proposed in [8] is based on a Kalman filter using an uncertain a priori stochastic model to represented the unknown force. This approach only requires to adjust one parameter which seems directly related to a trade-off between the resolution and the bandwidth of the force sensor. This paper adds to [8] an analysis of this trade-off. Its goal is to provide some tools to the end-user to help him adjusting the parameter. It begins by a short description of the force sensor. It is followed by a summary of the time-varying Kalman filter design presented in [8]. An analysis of the trade-off between resolution and bandwidth is developped using a steady-state Kalman filter. Finally, some new experimental results different from whose present in [8] are presented.

\section{FORCE SENSOR BASED ON DIAMAGNETIC LEVITATION}

The transducer used to estimate micro or nanoforces is an "heavy" macroscopic rigid seismic mass which is quite uncommon because of the unavoidable inertia effects of such designs. Some other prototypes using a macroscopic seismic mass can be found in [9] [10]. The seismic mass called maglevtube is a ten centimeters-long capillary tube stuck on two small magnets $M_{2}$ (see Figure 2). Its mass $m$ is around $70 \mathrm{mg}$. It is levitating passively around a given equilibrium state thanks to the diamagnetic levitation principle. When a force $F^{x}$ is applied to the tube tip along its longitudinal axis $\vec{x}$, the displacement $x$ obtained corresponds to a secondorder under-damped dynamic due to the magnetic springs embedded in the sensor and to the very small viscous friction of the air (the typical maglevtube damping ratio $\zeta$ is $6 \times 10^{-3}$ 


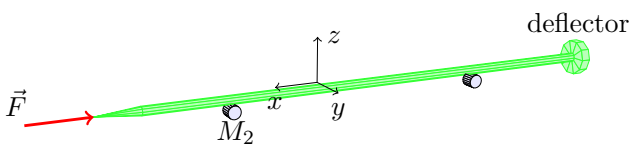

Fig. 2. Macroscopic force/displacement transducer (malevtube).

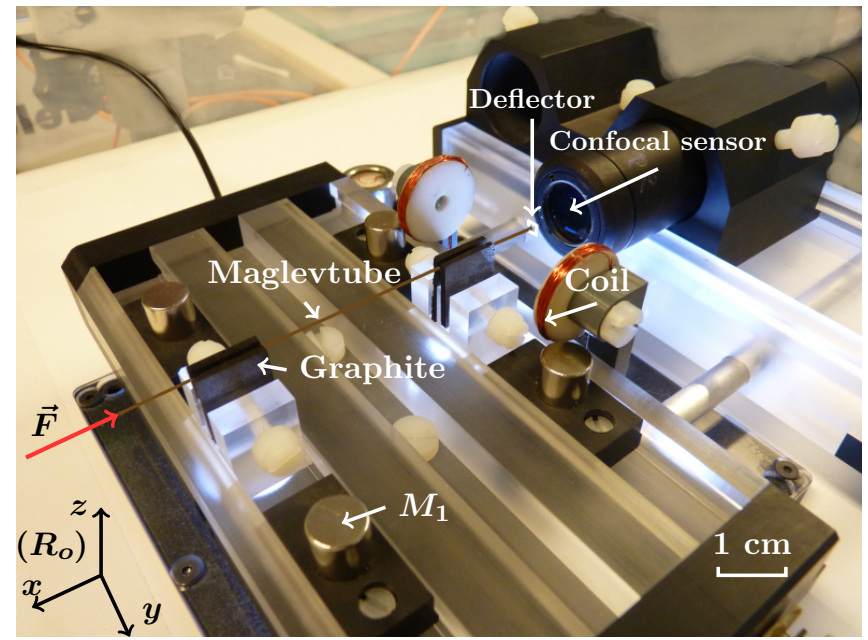

Fig. 3. Force sensor prototype.

and the $5 \%$ settling time is $20 \mathrm{sec}$ ). When the steady-state is reached, it comes:

$$
F^{x}=K_{m}^{x} x \quad K_{m}^{x}>0 .
$$

Typical magnetic stiffness $K_{m}^{x}$ is between $0.005 \mathrm{~N} / \mathrm{m}$ and $0.03 \mathrm{~N} / \mathrm{m}$. The measurement $m^{x}$ of $x$ is done with a confocal chromatic sensor manufactured by STIL SA. It is aimed at the deflector located at the rear of the maglevtube (see Figure 3). The sensor is practically linear even for millimetric displacements of the maglevtube [8] [11] which makes a large range of force measurement possible. Coils located at the rear of the sensor are only used during the sensor calibration to generate a zero input response.

\section{ForCE ESTIMATION WITH KALMAN FILTERING}

\section{A. A priori information for force estimation}

A priori information in the Figure 1 consists in modelling the second-order dynamic of the maglevtube and to merge it with an input force model to obtain an extended state-space model that will be used to estimate the input force. The input force model proposed in [8] is based on a stochastic Wiener process:

$$
\dot{F}(t)=\omega(t)
$$

$F(t)$ in (3) is the modelled real force $F^{x}(t)$ applied to the maglevtube tip along the $\vec{x}$ direction. $\omega(t)$ is a zero-mean infinite-variance white gaussian stochastic process representing the fact that the evolution of the force derivative is not known accurately. The autocorrelation function $\phi_{\omega, \omega}$ of this process is characterized by its power spectral density $W_{\dot{F}}$ :

$$
\phi_{\omega, \omega}(\tau)=W_{\dot{F}} \delta(\tau) \quad \forall \tau \in \mathbb{R}
$$

$W_{\dot{F}}$ is a scalar parameter to set by the end-user which influences in a given way the dynamic of the unknown force estimation. The extended state-space model combining the maglevtube dynamic and the force model is [8]:

$$
\begin{aligned}
\dot{X}^{e}(t) & =\mathcal{A} X^{e}(t)+\mathcal{M} \omega(t) \\
x(t) & =\mathcal{C} X^{e}(t)
\end{aligned}
$$

with $X^{e}(t)=\left[\begin{array}{lll}x & \dot{x} & F\end{array}\right]^{\top}$ and:

$$
\mathcal{A}=\left[\begin{array}{ccc}
0 & 1 & 0 \\
-\frac{1}{K_{m}^{x}} & -\frac{1}{K_{v}^{x}} & \frac{1}{m} \\
0 & 0 & 0
\end{array}\right] \quad \mathcal{M}=\left[\begin{array}{l}
0 \\
0 \\
1
\end{array}\right] \mathcal{C}=\left[\begin{array}{lll}
1 & 0 & 0
\end{array}\right]
$$

$x$ is the position of the centre of gravity of the maglevtube in the frame $R_{0}$ attached to its base (cf. Figure 3 ). $K_{m}^{x}$ is the magnetic stiffness and $K_{v}^{x}$ the viscous damping coefficient of the maglevtube that can be identified thanks to a zero input response (the calibration process is described in [8]). The discretization of (5) with a sampling period $T_{s}$ and a zero-order hold (zoh) on $\omega(t)$ leads to:

$$
\begin{aligned}
X_{k+1}^{e} & =\mathcal{F} X_{k}^{e}+\Omega_{k} \\
x_{k} & =\mathcal{C} X_{k}^{e}
\end{aligned}
$$

with $\mathcal{F}=e^{\mathcal{A} T_{s}}$ and:

$$
X_{k}^{e}=\left[\begin{array}{lll}
x_{k} & \dot{x}_{k} & F_{k}
\end{array}\right]^{\top} \quad \Omega_{k}=\left[\begin{array}{lll}
\omega_{k}^{x} & \omega_{k}^{\dot{x}} & \omega_{k}^{F}
\end{array}\right]^{\top}
$$

The zero-mean white gaussian process noise $\Omega_{k}$ characterizes uncertainties on $x_{k}, \dot{x}_{k}$ and $F_{k}$ due to the stochastic force model used and to the discretization of the maglevtube dynamic.

\section{B. Force estimation using a time-varying Kalman filter}

The input of the Kalman filter is the noisy measurement $m_{k}^{x}$ of the mavlevtube displacement $x_{k}$ :

$$
m_{k}^{x}=x_{k}+v_{k} \quad E\left[v_{k}^{2}\right]=R
$$

The zero-mean white gaussian noise $v_{k}$ with known variance $R$ is due to the confocal chromatic sensor. The output of the Kalman filter is $\hat{F}_{k}$ and its associated prediction-estimation stages are [8]:

$$
\begin{aligned}
& \hat{X}_{k \mid k-1}^{e}=\mathcal{F} \hat{X}_{k-1}^{e} \\
& P_{k \mid k-1}=\mathcal{F} P_{k-1} \mathcal{F}^{\top}+Q \\
& K_{k}=P_{k \mid k-1} \mathcal{C}^{\top}\left(\mathcal{C} P_{k \mid k-1} \mathcal{C}^{\top}+R\right)^{-1} \\
& \hat{X}_{k}^{e}=\hat{X}_{k \mid k-1}^{e}+K_{k}\left(m_{k}^{x}-\mathcal{C} \hat{X}_{k \mid k-1}^{e}\right) \\
& P_{k}=\left(I_{3 \times 3}-K_{k} \mathcal{C}\right) P_{k \mid k-1} \\
& \hat{F}_{k}=\mathcal{C}_{F} \hat{X}_{k}^{e}
\end{aligned}
$$

$\mathcal{C}_{F}=\left[\begin{array}{lll}0 & 0 & 1\end{array}\right]$ is the output matrix giving $\hat{F}_{k}$. This time-varying Kalman filter must be initialized before any force measurement (choice of $\hat{X}_{0}^{e}$ and $P_{0}$ ) and a numerical computation of $Q$ must be done each time $W_{\dot{F}}$ is changed by the end-user during the force estimation process [8]:

$$
\begin{aligned}
Q & =E\left[\Omega_{k} \Omega_{k}^{\top}\right]=W_{\dot{F}} \eta\left(T_{s}\right) \\
\eta\left(T_{s}\right) & =\int_{0}^{T_{s}} e^{\mathcal{A} t} \mathcal{M} \mathcal{M}^{\top} e^{\mathcal{A}^{\top} t} d t
\end{aligned}
$$




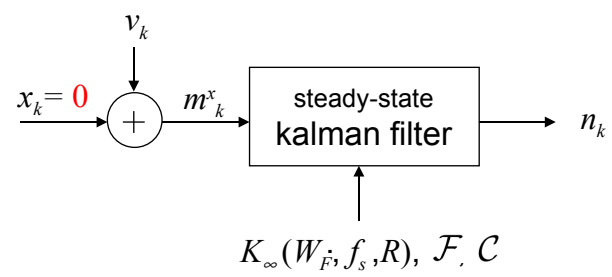

Fig. 4. Noise $n_{k}$ in estimation $\hat{F}_{k}$ due to the measurement noise $v_{k}$.

\section{ANALYSIS OF THE SENSOR RESPONSE}

\section{A. Trade-off between resolution and bandwidth}

In [8] the effect of the $W_{\dot{F}}$ parameter on the force estimation had been simply illustrated with a simulated step input force set to $100 \mathrm{nN}$. This effect is a trade-off between the resolution and the response time of the force estimation that can be qualitatively illustrated by the following Table:

\begin{tabular}{|c|c|c|}
\hline Force level & Force dynamic & Suitable $W_{\dot{F}}$ value \\
\hline high & small & small or high \\
high & high & high \\
small & small & small \\
small & high & - \\
\hline
\end{tabular}

This Table illustrates that it is impossible to have both a high resolution (to estimate correctly a small force) and a low response time (to estimate correctly a force with a high dynamic). A high resolution means a low noise in the force estimation and a low response time means a large bandwidth. To study more deeply this trade-off between resolution and bandwidth a steady-state Kalman filter is used to be independant of $P_{0}$ choice (transient response of the filter) and to manipulate only discrete-time-invariant statespace representation. Kalman gain $K_{k}$ is replaced by $K_{\infty}$ ( $K_{\infty}$ is obtained by solving the associated discrete Riccati equation and depends on $W_{\dot{F}}, f_{s}$ and $R$ ) and only equations (12) (15) (17) are used. The associated third-order state-space model of the steady-state Kalman filter with measurement $m_{k}^{x}$ as input and estimation $\hat{F}_{k}$ as output is obtained with (15) reported in (12) and using (17):

$$
\begin{aligned}
\hat{X}_{k+1 \mid k}^{e} & =A^{K} \hat{X}_{k \mid k-1}^{e}+B^{K} m_{k}^{x} \\
\hat{F}_{k} & =C^{K} \hat{X}_{k \mid k-1}^{e}+D^{K} m_{k}^{x}
\end{aligned}
$$

with

$$
\begin{array}{ll}
A^{K}=\mathcal{F}\left(I_{3 \times 3}-K_{\infty} \mathcal{C}\right) & B^{K}=\mathcal{F} K_{\infty} \\
C^{K}=\mathcal{C}_{F}\left(I_{3 \times 3}-K_{\infty} \mathcal{C}\right) & D^{K}=\mathcal{C}_{F} K_{\infty}
\end{array}
$$

\section{B. Force sensor resolution}

The additive gaussian noise $n_{k}$ presents in the estimation $\hat{F}_{k}$ is the consequence of the measurement noise $v_{k}$ (present in the input $m_{k}^{x}$ ) going through the kalman filter. Statistical properties of $n_{k}$ can be determined using the Kalman filter state-space model (20)-(21). As it is shown in the Figure 4 , thanks to additivity of linear systems, only input $v_{k}$ is considered instead of the sum $m_{k}^{x}=x_{k}+v_{k}$, which gives the following stochastic state-space model:

$$
\begin{aligned}
\hat{X}_{k+1 \mid k}^{e} & =A^{K} \hat{X}_{k \mid k-1}^{e}+B^{K} v_{k} \\
n_{k} & =C^{K} \hat{X}_{k \mid k-1}^{e}+D^{K} v_{k}
\end{aligned}
$$

Noise $v_{k}$ is the input and $n_{k}$ is the output. $X_{k \mid k-1}^{e}$ is a random state with mean $m_{k}$ and covariance matrix $S_{k}$ given by:

$$
\begin{aligned}
m_{k} & =E\left[\hat{X}_{k \mid k-1}^{e}\right]=\left(A^{K}\right)^{k} m_{0} \\
S_{k+1} & =E\left[\left(\hat{X}_{k+1 \mid k}^{e}-m_{k+1}\right)^{2}\right] \\
& =A^{K} S_{k} A^{K^{\top}}+B^{K} R B^{K^{\top}}
\end{aligned}
$$

with $R$ defined in (11). Taking into account the fact that $v_{k}$ is a zero-mean gaussian noise and that $\hat{X}_{k \mid k-1}^{e}$ and $v_{k}$ are independent at time $t_{k}$, the mean $\mu_{k}$ and variance $\Sigma_{k}$ of the gaussian noise $n_{k}$ in (25) are given by:

$$
\begin{aligned}
& \mu_{k}=E\left[n_{k}\right]=C^{K} m_{k}+D^{K} E\left[v_{k}\right]=C^{K} m_{k} \\
& \Sigma_{k}=E\left[\left(n_{k}-\mu_{k}\right)^{2}\right]=C^{K} S_{k} C^{K^{\top}}+D^{K} R D^{K^{\top}}
\end{aligned}
$$

Because of the sum $m_{k}^{x}=x_{k}+v_{k}$ on the input, any initial condition different from zero for the (deterministic) state $X_{k \mid k-1}^{e}$ will be associated to the input $x_{k}$. Thus, by additivity, it remains only a zero initial condition for the (stochastic) state $X_{k \mid k-1}^{e}$ associated to the input $v_{k}$ :

$$
X_{0 \mid-1}^{e}=\left[\begin{array}{lll}
0 & 0 & 0
\end{array}\right]^{\top}
$$

Because the initial condition for this stochastic state $X_{k \mid k-1}^{e}$ is always the previous one, it comes:

$$
m_{0}=0_{3 \times 1} \quad S_{0}=0_{3 \times 3}
$$

Notice that $m_{0}$ and $S_{0}$ only influence the transient evolution of $n_{k}$ at the beginning of the measurement. With (26) the mean $m_{k}$ is always equal to zero and with (28) the statistical properties of $n_{k}$ are given by:

$$
\begin{aligned}
& \mu_{k}=0 \quad \forall k \\
& \Sigma_{k}=C^{K} S_{k} C^{K^{\top}}+D^{K} R D^{K^{\top}}
\end{aligned}
$$

The $99 \%$ confidence interval associated to (33) is plotted with red lines in the Figure 5 for a $100 \mathrm{nN}$ step force. The sampling frequency $f_{s}$ is set to $100 \mathrm{Hertz}$ in this simulation. The mean in the Figure corresponds to the value of $\hat{F}_{k}$ when there is no measurement noise $v_{k}$. This unknown value belongs to the confidence interval with a 0.99 probability. To avoid any misinterpretation, it doesn't mean that the real force $F^{x}(t)$ belongs to this interval (obviously, this is not the case during the beginning of the step transition), but only that $\hat{F}_{k}$ should belong to it if they were no measurement noise. The associated standard deviation $\left(\Sigma_{k}\right)^{0.5}$ is an image of the resolution (or the SNR ratio) of the sensor. It tends to $\left(\Sigma_{\infty}\right)^{0.5}$ in a few sampling times $t_{k}$ (see the first 0.05 sec in the Figure 5). This value $\left(\Sigma_{\infty}\right)^{0.5}$ can be given to the end-user to adjust $W_{\dot{F}}$. Bigger is $W_{\dot{F}}$ and bigger is $\Sigma_{\infty}$ (see Figure 6), thus lower is the resolution of the sensor. Variance $\Sigma_{k}$ has no connection to the force sensor accuracy. In steady-state, the accuracy only depends on the calibration of $K_{m}^{x}$. 


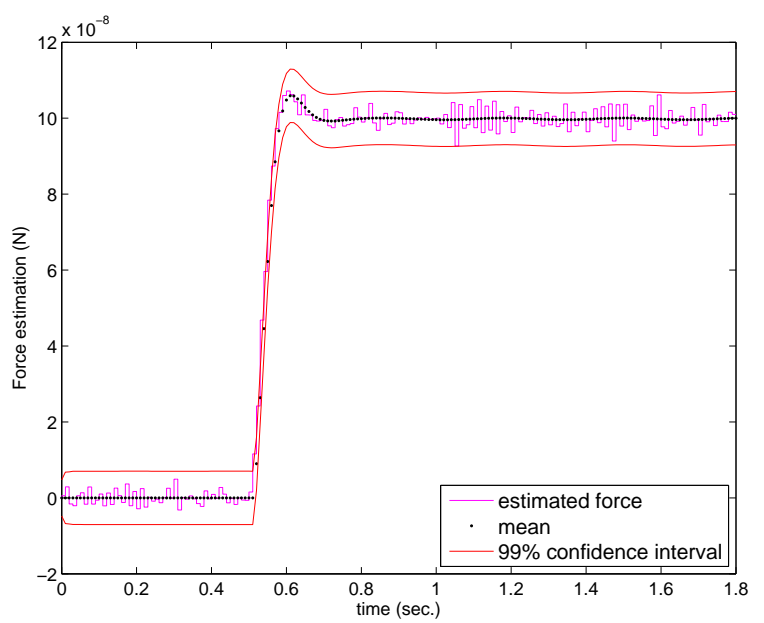

Fig. 5. $99 \%$ confidence interval $\left( \pm 3 \sqrt{\Sigma_{k}}\right)$ on the force estimation with $W_{\dot{F}}=10^{-15} \mathrm{~N}^{2} / \mathrm{Hz}$ and $f_{s}=100 \mathrm{~Hz}$.

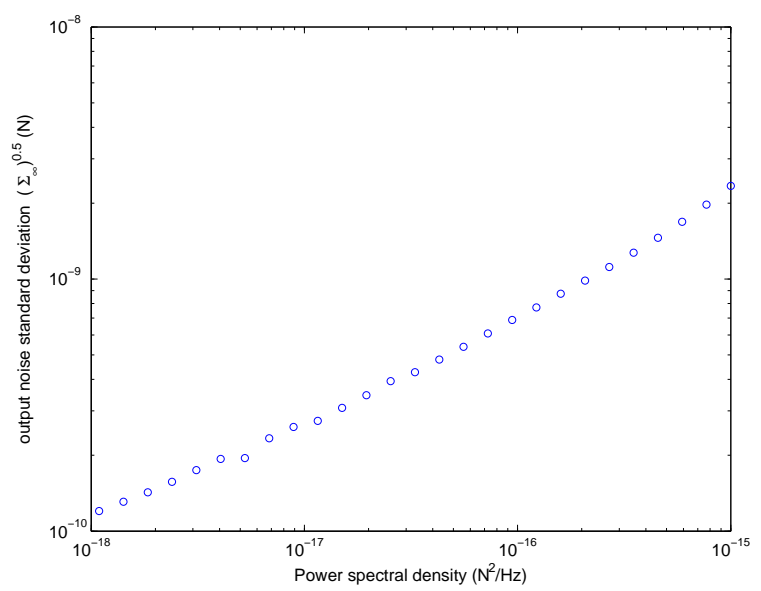

Fig. 6. Standard deviation $\sqrt{\Sigma_{\infty}}$ versus $W_{\dot{F}}$ for $f_{s}=100 \mathrm{Hertz}$ and $R=$ $1.44 \times 10^{-16} \mathrm{~m}^{2}$. Small irregularities are mainly due to numerical errors during the solving of discrete Riccati equation to obtain $K_{\infty}\left(W_{\dot{F}}, f_{s}, R\right)$.

\section{Force sensor bandwidth}

The bandwidth of the force estimation depends on the harmonic transfer between the input force $F^{x}(t)$ and the output estimation $\hat{F}_{k}$. Because time description is continuous for the input and discrete for the output, this transfer is difficult to model. Thus, it will be determined with only a discrete time description: the second-order transfer from $F^{x}(t)$ to $x(t)$ (dynamic of the maglevtube) is discretized with a zoh added on $F^{x}(t)$ with a sampling period $T_{s}$. This will leads to a slightly erroneous result when a pure sinusoidal input $F^{x}(t)$ is applied on the maglevtube. The global discrete state representing the maglevtube state and the Kalman filter state is:

$$
\mathcal{X}_{k}=\left[\begin{array}{c}
X_{k} \\
\hat{X}_{k \mid k-1}^{e}
\end{array}\right]
$$

with $X_{k}=\left[\begin{array}{ll}x_{k} & \dot{x}_{k}\end{array}\right]^{\top}$ the maglevtube state associated to a classical $2^{\text {nd }}$ order state-space representation:

$$
\begin{gathered}
X_{k+1}=A_{d} X_{k}+B_{d} F_{k}^{x} \\
m_{k}^{x}=C_{d} X_{k}+v_{k} \\
A_{d}=\exp \left(\left[\begin{array}{ll}
\mathcal{A}_{11} & \mathcal{A}_{12} \\
\mathcal{A}_{21} & \mathcal{A}_{22}
\end{array}\right] T_{s}\right) \quad C_{d}=\left[\begin{array}{ll}
1 & 0
\end{array}\right] \\
B_{d}=\int_{0}^{T} \exp \left(\left[\begin{array}{ll}
\mathcal{A}_{11} & \mathcal{A}_{12} \\
\mathcal{A}_{21} & \mathcal{A}_{22}
\end{array}\right] t\right) d t\left[\begin{array}{l}
\mathcal{A}_{13} \\
\mathcal{A}_{23}
\end{array}\right]
\end{gathered}
$$

Then (36) is merged in (20) and (21):

$$
\begin{aligned}
\hat{X}_{k+1 \mid k}^{e} & =A^{K} \hat{X}_{k \mid k-1}^{e}+B^{K} C_{d} X_{k}+B^{K} v_{k} \\
\hat{F}_{k} & =C^{K} \hat{X}_{k \mid k-1}^{e}+D^{K} C_{d} X_{k}+D^{K} v_{k}
\end{aligned}
$$

and (34), (39), (40), (35) leads to the following global statespace representation of the force sensor with $F_{k}^{x}$ and $v_{k}$ as input and $\hat{F}_{k}$ as output:

$$
\begin{gathered}
\mathcal{X}_{k+1}=A_{g} \mathcal{X}_{k}+B_{g}\left[\begin{array}{c}
F_{k}^{x} \\
v_{k}
\end{array}\right] \\
\hat{F}_{k}=C_{g} \mathcal{X}_{k}+D_{g}\left[\begin{array}{c}
F_{k}^{x} \\
v_{k}
\end{array}\right]
\end{gathered}
$$

with

$$
\begin{array}{rlr}
A_{g} & =\left[\begin{array}{cc}
A_{d} & 0_{2 \times 3} \\
B^{K} C_{d} & A^{K}
\end{array}\right] & B_{g}=\left[\begin{array}{cc}
B_{d} & 0_{2 \times 1} \\
0_{3 \times 1} & B^{K}
\end{array}\right] \\
C_{g}=\left[\begin{array}{ll}
D^{K} C_{d} & C^{K}
\end{array}\right] & D_{g}=\left[\begin{array}{ll}
0 & D^{K}
\end{array}\right]
\end{array}
$$

Setting noise $v_{k}$ to zero to study only the impact of $F_{k}^{x}$ input gives the global harmonic transfer $H_{g}(\omega)$ between the force to measure and the force estimated:

$$
H_{g}(\omega)=\frac{\hat{F}\left(e^{j \omega}\right)}{F^{x}\left(e^{j \omega}\right)}=C_{g}\left(e^{j \omega} I_{5 \times 5}-A_{g}\right)^{-1}\left[\begin{array}{c}
B_{d} \\
0_{3 \times 1}
\end{array}\right]
$$

The Figure 7 shows the global frequency response of the sensor for different values of $W_{\dot{F}}$ when $A_{d}$ and $B_{d}$ are perflectly known (perfect calibration). Sampling frequency $f_{s}$ is set to 1000 Hertz to minimize the effect of the zoh in (35). The step force response (with $v_{k}$ set to zero) is also plotted to see the matching between response time and frequency response of the estimation. Higher is $W_{\dot{F}}$ and larger is the force bandwidth of the sensor. As it is shown, the force bandwidth can be extended beyond the $-3 \mathrm{~dB}$ cutoff frequency of the maglevtube (around $4 \mathrm{~Hz}$ ). This frequency response can be provided to the end-user to help him adjusting $W_{\dot{F}}$. The Figure 8 shows the global amplitude frequency response when the calibration is not perfectly accurate. The values chosen are those given in [8]. The scale on Y-axis is linear contrary to the one in the Figure 7(a). Force measurement around the resonant frequency of the maglevtube can lead to non negligible errors.

\section{EXPERIMENTAL MEASUREMENTS}

\section{A. Disturbance forces}

$\hat{F}_{k}$ is an estimation of $F^{x}(t)$ that represents the sum of all external forces applied on the Maglevtube. The Figure 9 shows $\hat{F}_{k}$ when no external force is artificially applied 


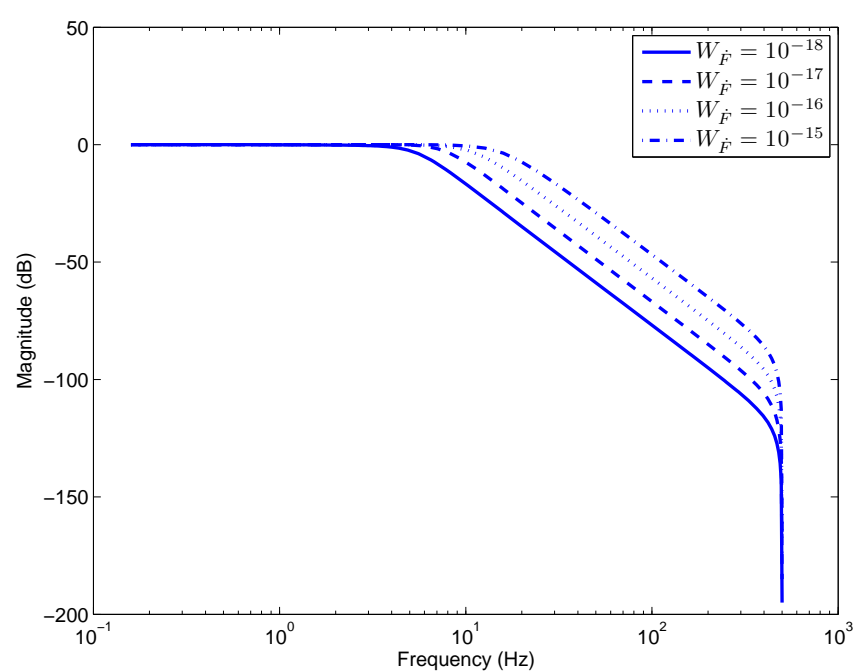

(a) Amplitude of $H_{g}\left(e^{j \omega}\right)$.

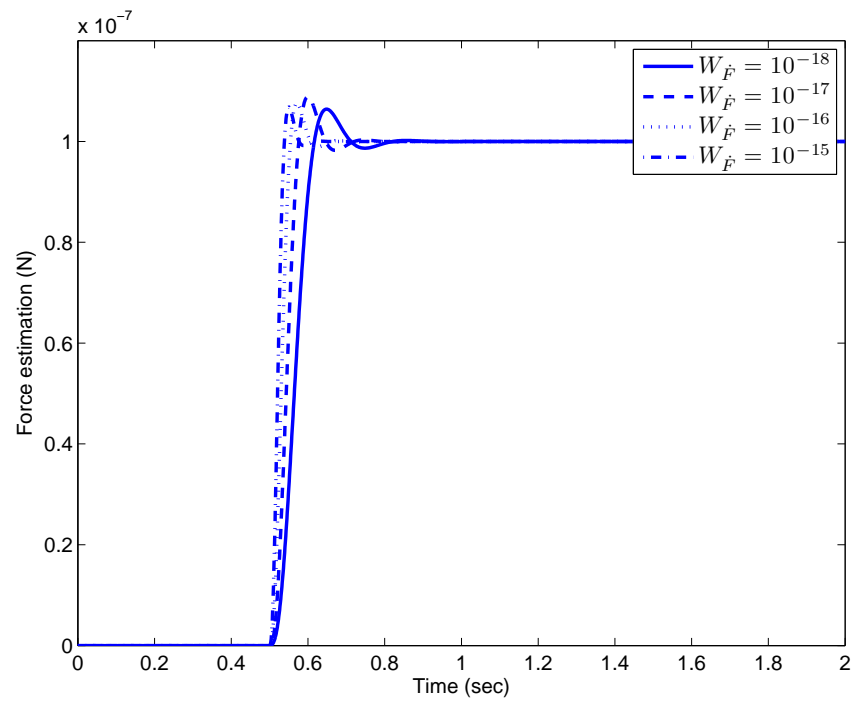

(b) Step force response with noise $v_{k}$ set to zero.

Fig. 7. Impact of $W_{\dot{F}}$ on the global frequency response $H_{g}\left(e^{j \omega}\right)$ and the response time of the sensor $\left(f_{s}=1000 \mathrm{Hertz}\right.$ and accurate calibration).

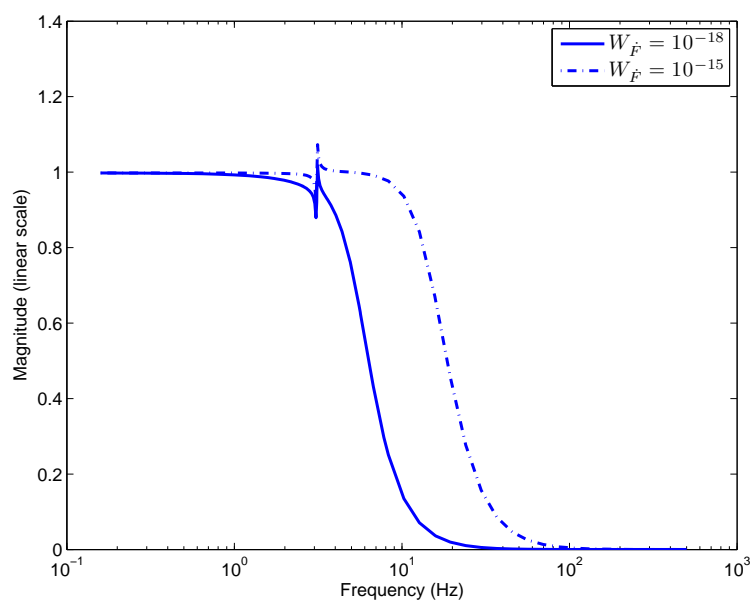

Fig. 8. Global amplitude frequency response $H_{g}\left(e^{j \omega}\right)$ with the calibration used.

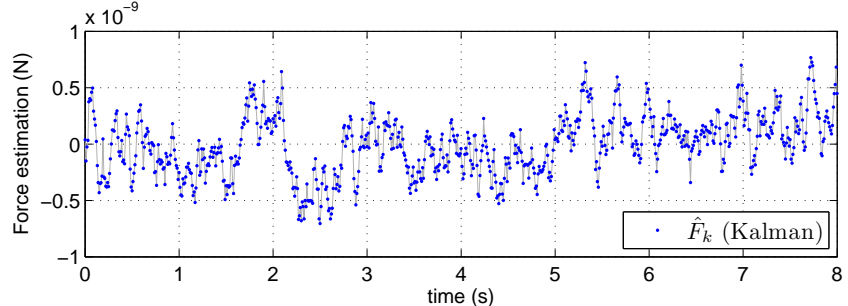

Fig. 9. Measurement of the external disturbance force in the afternoon.

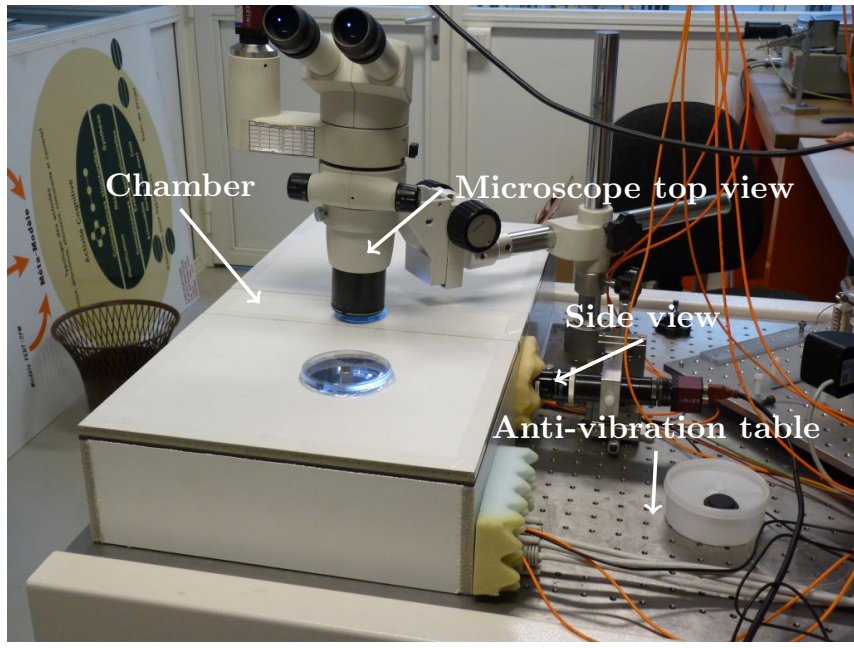

Fig. 10. Force sensor enclosed in a chamber on a antivibration table.

on the Maglevtube. In this case $\hat{F}_{k}$ is an estimation of all the external disturbance forces applied on the maglevtube along $\vec{x}$ by the environmental noise (see Figure 1). Because of the maglevtube $-3 \mathrm{~dB}$ cutoff frequency at 4 Hertz, the most predominant disturbance forces are due to the seismic low frequency vibrations of the sensor base (that generates a vibration of the magnetic field) and to air vibration in infrasound frequencies that generates an acoustic force which is mainly applied on the rear deflector. An extreme care must be taken to reduce as much as possible this environmental noise (sensor enclosed in a chamber put on a antivibration table inside a small room with massive concrete ground floor shown in the Figure 10). Maximum disturbance force amplitude is inferior to $1 \mathrm{nN}$ and unbiased standard deviation is inferior to $0.3 \mathrm{nN}$. The figure 11 illustrates the effect of a modification of $W_{\dot{F}}$ during the measurement of a real force varying very slowly around $255 \mathrm{nN}$.

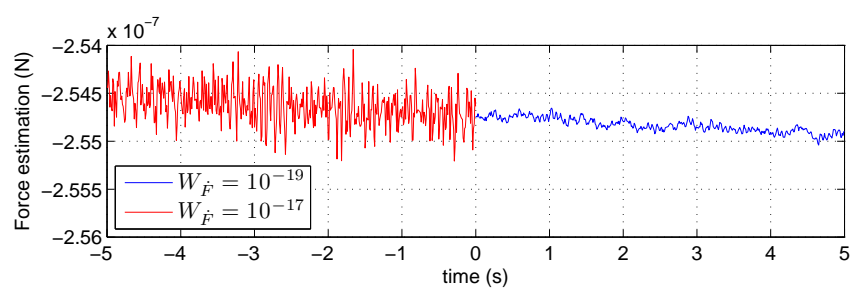

Fig. 11. Modification of $W_{\dot{F}}$ value during a force measurement. 


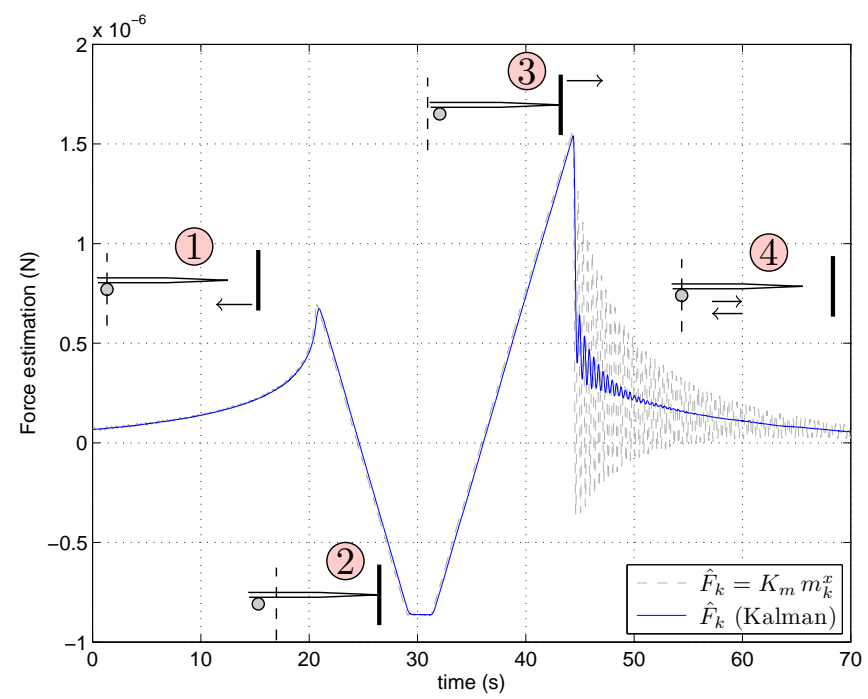

Fig. 12. Experimental pull-off force measurement with non-contact attractive force also applied on the Maglevtube.

\section{B. Pull-off force measurement}

The Figure 12 shows the evolution of the force $\hat{F}_{k}$ during a pull-off force measurement. A planar material is pushed against a micro-sphere stuck at the maglevtube tip (loading stages 1 and 2) and then moved back (unloading stage 3) until the contact is broken between the material and the sphere. A non-contact attractive force (electrostatic force) is also applied on the maglevtube sphere before the loading, during the loading/unloading and after the contact break. The sphere used is an electrically charged macroscopic sphere (one millimetre diameter, Silicon nitride $S i_{3} N_{4}$ ). The planar material is a cover glass (insulating material). The atmosphere relative humidity was low (no meniscus was observed during the pull-off measurement). The oscillations after the contact break represent the evolution of the non-contact force applied on the sphere when the cover glass is moved back. As the sphere moves towards and backwards the cover glass (because of the maglevtube big inertia) the non-contact force is oscillating. The mean of these oscillations has the same mirroring shape than the non-contact force before the loading. Because the speed of the planar material is opposite in both cases, this probably means that the distribution of charges in both material remained the same during all the force measurement. Equation (2) gives a bad estimation because the dynamic of the maglevtube is not taken into account. Kalman estimation (17) gives a better result with a shorter and smaller oscillating dynamic response.

\section{CONCLUSION}

Despite the inertia of their mass that should be a great handicap for time-varying force measurement, nanoforce sensors based on a rigid macroscopic seismic mass are not disqualified for low frequency force measurements compared to classical designs based on microscopic elastic cantilevers like AFMs. The design proposed in this paper is based on diamagnetic levitation and stiffness as low as $0.005 \mathrm{~N} / \mathrm{m}$ can be reached. Nevertheless, this design imposes some constraints like the limited bandwidth and the deconvolution of the mass displacement to take care of the behaviour imposed by the mass inertia. This deconvolution is done with a discrete Kalman filter that is using a discretized Wiener process to model the unknown input force. This processing requires the adjustment of a single parameter $W_{\dot{F}}$ which directly adjusts a trade-off between the resolution (standard deviation) of $\hat{F}_{k}$ and the response time of the estimation. The resolution and frequency response of the sensor can also be plotted to make this choice easier. This deconvolution method is computationally cheap (third-order IIR filter if a steady-state Kalman filter is chosen). Response time shorter than 0.1 second can be reached despite the very long settling time of the transducer (20 seconds) and its underdamping. Compared to a simple low-pass filter added on the displacement measurement that will inevitably restrict the bandwidth, the force bandwidth can be extended reasonably four times higher than the displacement bandwidth. The main drawback of this open-loop design is its sensitity to unwanted external disturbant forces. Extreme care must be taken to reduce as much as possible this environmental noise thanks to an antivibration table and a closed chamber.

\section{ACKNOWLEDGMENT}

This work was supported by the French National Research Agency under STIL $\mu$ FORCE ANR-07-ROBO-0005 contract.

\section{REFERENCES}

[1] P. Rougeot, S. Régnier, and N. Chaillet, "Forces analysis for micromanipulation," Proceedings 2005 IEEE international symposium on computational intelligence in robotics and automation, espoo, Finland, pp. 105-110, june 2005 .

[2] N. Kato, I. Suzuki, H. Kikuta, and K. Iwata, "Force-balancing microforce sensor with an optical-fiber interferometer," Review of scientific instruments, vol. 68, pp. 2475-2478, juin 1997.

[3] F. Arai, T. Sugiyama, T. Fukuda, H. Iwata, and K. Itoigawa, "Micro tripaxial force sensor for 3d bio-micromanipulation," In IEEE International Conference on Robotics and Automation (ICRA), 1999.

[4] F. Beyeler, S. Muntwyler, and B. J. Nelson, "A six-axis mems forcethorque sensor with micro-newton and nano-newtonmeter resolution," Journal of Microelectromechanical Systems, vol. 18, pp. 433-441, 2009.

[5] Y. Shen, N. Xi, and W. J. li, "Contact and force control in microassembly," In IEEE 5th International Symposium on Assembly and Task Planning (ISATP), pp. 60-65, 2003.

[6] A. Cherry, E. Piat, and J. Abadie, "Estimation robuste par synthèse h2 de micro- et nanoforce à l'aide de ressorts magnétiques actifs," JESA, vol. 44, no. 6, pp. 631-659, 2010.

[7] M. Rakotondrabe and P. Lutz, "Force estimation in a piezoelectric cantilever using the inverse-dynamics-based uio technique'," IEEE International Conference on Robotics and Automation (ICRA), pp. 2205-2210, 2009.

[8] E. Piat, J. Abadie, and S. Oster, "Nanoforce estimation with kalman filtering applied to a force sensor based on diamagnetic levitation," in IEEE International Conference on Intelligent Robots and Systems (IROS), San Francisco, Sept. 25-30 2011, pp. 39-44.

[9] Y. Fujii, "Method for generating and measuring the micro-newton level forces," Mechanical Systems and Signal Processing, vol. 20, pp. 13621371, 2006.

[10] A. Cherry, E. Piat, and J. Abadie, "Analysis of a passive microforce sensor based on magnetic springs and upthrust bouoyancy," Sensors \& Actuators: A. Physical, vol. 169, pp. 27-36, 2011.

[11] J. Abadie, E. Piat, S. Oster, and M. Boukallel, "Modeling and experimentation of a passive nanoforce sensor based on diamagnetic levitation," Sensors \& Actuators: A. Physical, vol. 173, pp. 227-237, 2012. 